\title{
Letale Komplikation eines Kolon-Kontrasteinlaufs durch Fehllage des Einlaufschlauchs in der Vagina
}

\author{
H. T. Haffner und M. Graw \\ Institut für Gerichtliche Medizin (Direktor: Prof. Dr. med. Dipl.-Phys. H.-D. Wehner) der Universität Tübingen
}

Bei einer 68 Jahre alten Frau sollte wegen rezidivierender krampfartiger Unterbauchbeschwerden eine Kolon-Kontrastdarstellung durchgeführt werden. In der Anfangsphase der Untersuchung sah der verantwortliche Radiologe bei der Durchleuchtungskontrolle einen Kontrastmittelstopp im Bereich des eingeführten Schlauchendes. Unter der Annahme eines Passagehindernisses erhöhte er den Kontrastmittel-Einlaufdruck. Daraufhin kam es plötzlich zu einem therapieresistenten Schock, und die Patientin starb. Die Obduktion ergab, daß es bei unbemerkter Fehllage des Einlaufschlauchs in der Scheide zu einem Vaginalwandriß mit subperitonealer Kontrastmittelinfiltration gekommen war. Radiologisch und histologisch konnte eine Kontrastmittelembolie in die Lunge nachgewiesen werden. Als Todesursache war das Zusammenwirken der Embolie mit einem peritoneal ausgelösten vagalen Schockzustand anzunehmen.

\section{Fatal complication of a barium enema of the colon resulting from unrecognized placement of the infusion catheter in the vagina}

A 68-year-old woman was to have a barium enema of the colon to discover the cause of recurrent cramp-like symptoms in the lower abdomen. At the beginning of the examination the responsible radiologist saw, during a fluoroscopic check, that the contrast medium had not advanced beyond the region of the infusion catheter tip. Believing this to be due to blockage in the catheter he increased the infusion pressure. Suddenly the patient went into treatment-resistant shock and died. The autopsy revealed that the infusion catheter had by mistake been placed into the vagina. This had caused a tear in the vagina with resulting subperitoneal contrast-medium infiltration. Embolization of contrast medium to the lung was demonstrated both histologically and radiologically. The cause of death was the combined effect of the embolism and peritoneally induced vagal shock.
Die Einführung eines Einlaufschlauchs in das Rektum gilt als einfache ärztliche Maßnahme, die häufig an Pflegepersonal delegiert wird. In der Regel ist dem Durchführenden in erster Linie die Gefahr einer Darmwandperforation als seltene Komplikation bewußt. Bei allzu sorgloser Handhabung besteht jedoch bei Frauen die Gefahr einer weiteren Komplikation, deren Auswirkungen nicht minder dramatisch sein können: die unbemerkte Fehlplazierung des Einlaufschlauchs in die Vagina.
Dtsch. med. Wschr. 118 (1993), 181-184

(c) Georg Thieme Verlag Stuttgart · New York

\section{Kasuistik}

Die 68 Jahre alte Frau, die bis dahin weitgehend gesund gewesen war, wurde wegen rezidivierender, starker Krämpfe im linken Unterbauch und Stuhlunregelmäßigkeiten stationär aufgenommen. Unter anderem war eine Kolon-Kontrastdarstellung vorgesehen.

\section{Untersuchungsablauf}

Der durchführende Arzt beschrieb, er habe nach gezielter Anamnese, Durchleuchtung im Stehen und digitaler Austastung des Rektums der Patientin den Ablauf der Untersuchung erklärt. Danach habe er ihr in Linksseitenlage unter Spreizung der Gesäßbacken und unter optischer Kontrolle 
den Einlaufschlauch eingeführt und mittels Ballon blockiert. Unter Durchleuchtungskontrolle habe er Kontrastmittel (Bariumsulfat) einlaufen lassen, das sich nur um das Schlauchende angesammelt habe. Den Kontrastmittelstopp habe er auf ein Passagehindernis zurückgeführt und deshalb den Kontrastmittelbehälter unter leichten Druck gesetzt. Die Patientin, die zunächst lediglich Stuhldrang angegeben hatte, habe dann plötzlich über starke Unterbauchschmerzen geklagt. Dann sei sie bewußtlos geworden, gleichzeitig sei der Einlaufschlauch aus der Körperöffnung geglitten. Puls und Blutdruck seien nicht mehr meßbar gewesen, die sofort eingeleitete halbstündige Reanimation habe nicht zum Erfolg geführt.

Vom leitenden Radiologen wurde die vorgesehene Untersuchung zur Klärung des letalen Ablaufs postmortal wiederholt. Das Rektum war ungehindert darstellbar und wies keine pathologischen Befunde auf. Daraufhin wurde von der Klinik die zuständige Staatsanwaltschaft über den Verdachtsfall eines nicht-natürlichen Todes informiert.

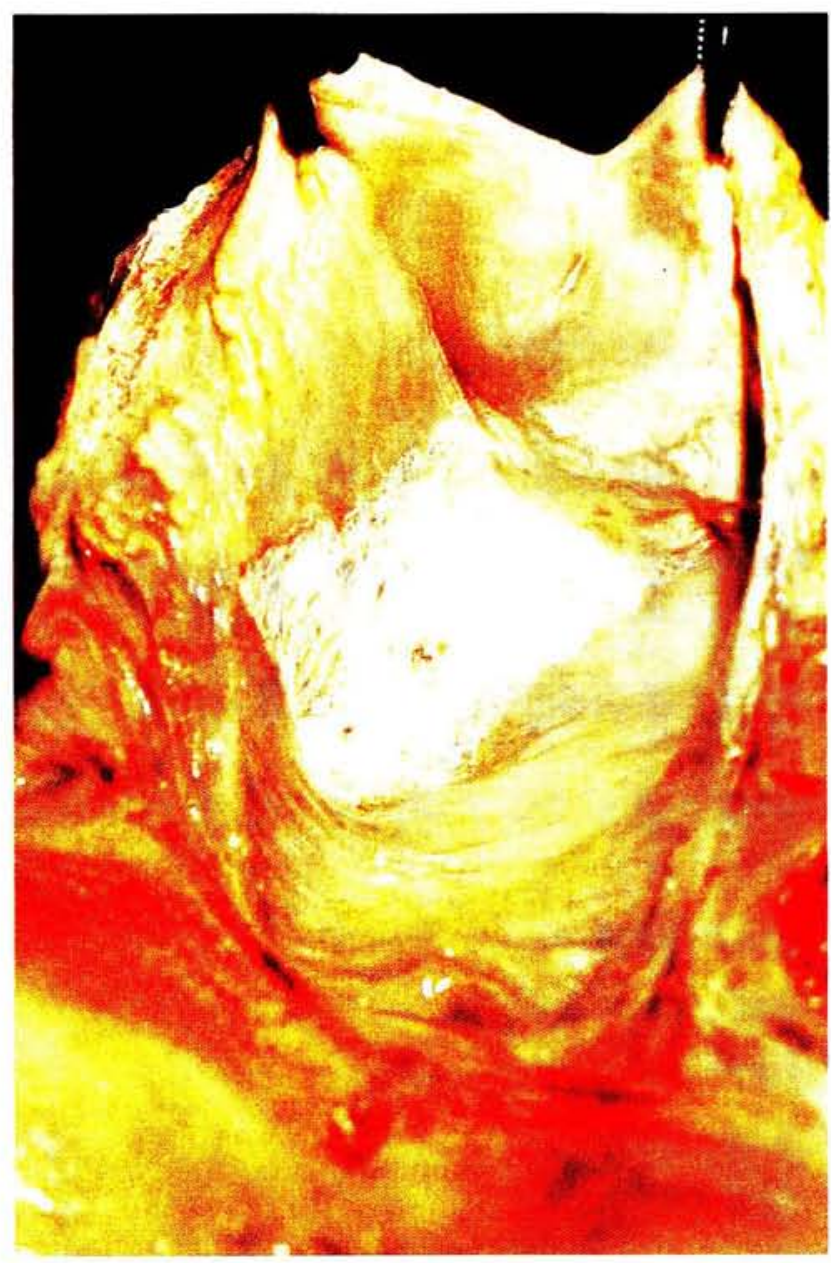

Abb. 1 Diagnonal verlaufender, mit Kontrastmittel durchtränkter Aufriß der Vaginalschieimhaut (Aufsicht auf die dorsale Scheidenwand, rechts oben von Klemmen flankiert der Muttermund).

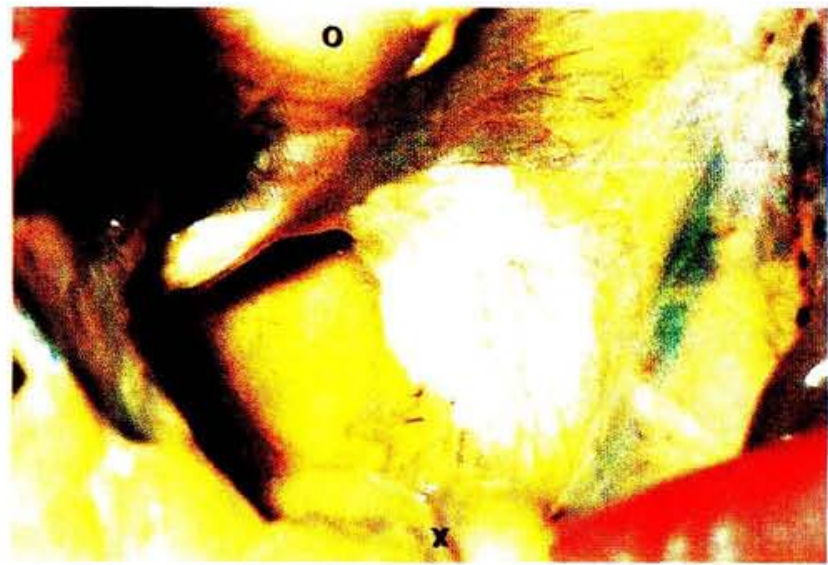

Abb. 2 Subperitoneale Kontrastmitteldepots (Einblick in den Douglasraum, $\mathrm{O}=$ dorsale Uterusfläche, $\mathrm{X}=$ ventrale Sigmoidfläche).

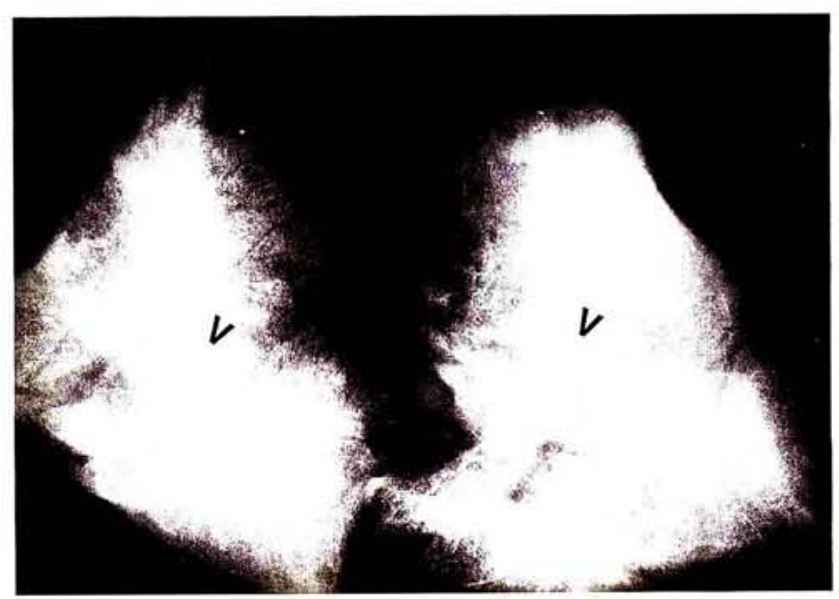

Abb. 3 Radiologischer Kontrastmittelnachweis in den hilusnahen Gefäßen beider Lungenflügel (Pfeile) (Aufnahme nach Organentnahme, keine regelrechte Topographie).



Abb. 4 Kleine Kontrastmittelemboli (Pfeile) in einem peripheren Lungengefäß. Hämatoxylin-Eosin, $400: 1$. 


\section{Obduktionsbefunde}

Bei der gerichtlichen Leichenöffnung wurde der Austritt von Kontrastmittel aus dem After und von blutig durchsetztem Kontrastmittel aus der Scheide festgestellt. Das Rektum war noch mit Kontrastmittel gefüllt. Colon ascendens und transversum waren gasgebläht und dilatiert, Colon descendens und Sigmoid spastisch eng gestellt. Die Darmwand zeigte keine pathologischen Befunde, so daß von einem Colon irritable als Ursache der Beschwerden ausgegangen werden mußte. Auch die Vagina war mit Kontrastmittel gefüllt. Nach Säubern zeigte sich in der dorsalen Scheidenschleimhaut ein $12 \mathrm{~cm}$ langer, diagonal verlaufender mit Kontrastmittel belegter und im Randbereich unterminierter Aufriß (Abbildung 1). Bei Inspektion der Bauchhöhle fielen zwei subperitoneal gelegene, durchscheinende Kontrastmitteldepots von bis $5 \mathrm{~cm}$ Durchmesser in Höhe der Plica rectouterina auf. Darüber hinaus bestand hier regional eine vermehrte peritoneale Gefäßinjektion (Abbildung 2). Das Blut in der rechten Herzkammer sowie in den Hauptästen der Pulmonalarterien war von einer feingrießeligen weißlichen Substanz diskret durchmischt. Eine Röntgenaufnahme der Lungen nach Organentnahme ließ Kontrastmittelansammlungen im Hilusbereich erkennen (Abbildung 3). Es bestand lediglich eine diskrete Dilatation beider Herzhöhlen. Eine akute Blutstauung der Bauchorgane war nicht vorhanden.

Histologisch konnten nur vereinzelt Kontrastmittelemboli in den peripheren LungengefäBen nachgewiesen werden, die sich zu den Kontrastmittelinfiltrationen des subperitonealen Bindegewebes identisch darstellten (Abbildung 4). Als Folge des Schocks zeigten sich in den Lungen eine Hyperämie sowie eine Fett- und Knochenmarksembolie. In den übrigen Organen (Gehirn, Herz, Leber, Milz, Nieren) waren keine Kontrastmittelemboli nachweisbar.

Als Todesursache wurde ein akutes Herz-Kreislauf-Versagen angegeben bei Zusammenwirken eines peritoneal ausgelösten reflektorischen vagalen Schocks und einer Kontrastmittelembolie in die Lungen.

\section{Diskussion}

Die vorgestellte Kasuistik ist keineswegs ein exotischer Einzelfall; über ähnliche Fälle berichteten auch andere Autoren (1-5). Es handelte sich jeweils um Kontrastmitteleinläufe bei älteren Patientinnen, die trotz vorangegangener Aufklärung über den Gang der Untersuchung, möglicherweise aus Schamgefühl oder autoritätsbedingter Hilflosigkeit, auf die Fehlplazierung des Einlaufschlauchs nicht aufmerksam machten. Das Darmrohr war, mit Ausnahme des vorgestellten Falles, von einer Röntgenassistentin oder Krankenschwester eingeführt worden; angeblich durchgeführte Kontrollen deckten die Fehllage nicht auf. Bei der Kontrastmittelinsufflation kam es zu überwiegend doppelseitigen lateralen Vaginalwandaufrissen mit retroperitonealen, in dem von Mattig und Mitarbeitern (5) geschilderten Fall nach Bauchfellruptur auch zu intraperitonealen Kontrastmittelansammlungen.

Die weiteren pathophysiologischen Abläufe waren in den publizierten Fällen teilweise unterschiedlich. Geipel (3) sowie Bayer und Mitarbeiter (1) berichteten über längere Überlebenszeiten (3 Tage bzw. 15 Stunden). Als Todesursache stellten sie eine durch fortwährende Kontrastmitteleinschwemmung aus den retroperitonealen Depots protrahiert verlaufende Lungenembolie mit Rechtsherzversagen fest. In den von Bonte und Sprung (2) sowie von Mattig und Lignitz (4) geschilderten Fällen trat der Tod wie auch bei der von uns vorgestellten Patientin innerhalb von wenigen Minuten ein. Bonte und Sprung (2) konnten bei der Obduktion ein großes verletztes Gefäß des Plexus uterovaginalis darstellen, über das es zu einer fulminanten Kontrastmittelembolie gekommen war. Mattig und Lignitz (4) dagegen gaben ein nicht näher differenziertes Schockgeschehen als Todesursache an; eine Embolie hielten sie für unwahrscheinlich.

Auch im vorgestellten Fall muß aufgrund der morphologischen Befunde eine Embolie als alleinige Todesursache in Frage gestellt werden. Zwar steht außer Zweifel, daß es zu einer Kontrastmittelembolie in die Lungen kam. Das Ausmaß der Embolie war jedoch sowohl radiologisch als auch histologisch im Vergleich mit den anderen bekannten Fällen zu gering, um ohne weiteres eine hämodynamisch ausreichende Wirksamkeit zu unterstellen; prädisponierende Schäden des Herz-KreislaufSystems bestanden nicht. Ferner fehlte das klassische Bild des akuten Rechtsherzversagens. Da durch das Kontrastmittel Bariumsulfat, eine chemisch inerte Substanz, weder allergische noch akut toxische Reaktionen zu befürchten sind, ist als zusätzlicher für den Tod bedeutsamer Faktor in erster Linie ein vagaler Reflex infolge der mechanischen Peritonealreizung zu diskutieren. Nach Tschirren (6) treten durch derartige vegetative Reize zwar unter normalen Bedingungen keine ernsthaften Komplikationen auf; die Anfälligkeit für vegetative Reize ist jedoch im Zustand der Hypoxie so stark erhöht, daß Refleximpulse dann auch zu einem Herzstillstand führen können.

Die Übersicht über die aus der Literatur bekannten Fälle zeigt demnach, daß es in der Folge der versehentlichen retroperitonealen Bariumsulfat-Instillationen zu unterschiedlichen pathophysiologischen Abläufen kommen kann: Wird bei der Gewebstraumatisierung ein größeres Gefäß eröffnet, tritt eine fulminante Embolie mit Rechtsherzversagen ein (2). Wird dagegen kein größeres Gefäß verletzt, so ist mit einer protrahiert verlaufenden Embo- 
lisierung zu rechnen, die aus den im traumatisierten Gewebe liegenden Kontrastmitteldepots gespeist wird (1, 3). Bei starker mechanischer Reizung des Peritoneums durch die subperitonealen Kontrastmittelansammlungen oder durch peritoneale Perforationen bei gleichzeitiger Hypoxie durch die in Gang gekommene Kontrastmittelembolisierung der Lunge kann schließlich ein vagaler Reflex zum Herz-Kreislauf-Stillstand führen $(4,5)$. Diesen Pathomechanismus vermuten wir auch im vorgestellten Fall.

Bei der gerichtsmedizinischen Begutachtung bereiten Fehlplazierungen des Einlaufschlauchs in die Vagina keine größeren Probleme. $\mathrm{Zu}$ Beginn der Untersuchung muß das Darmrohr unter optischer Kontrolle in den Enddarm eingeführt werden. Dabei sind Verwechselungen der Körperöffnungen kaum möglich. Insofern sind Fehlplazierungen des Einlaufschlauchs in die Vagina in der Regel als Verletzungen der ärztlichen Sorgfaltspflicht anzusehen. Entsprechend wurde auch im vorgestellten Fall der beschuldigte Arzt wegen fahrlässiger Tötung zu einer Geldstrafe von 12000 DM verurteilt.

\section{Literatur}

1 Bayer, H. P., F. Bühler, J. Ostermeyer: Zur Verteilung interstitiell und parenteral applizierten Bariumsulfats im Organismus. Z. Rechtsmed. 74 (1974), 207.

2 Bonte, W., R. Sprung: Letale Bariumsulfatembolie nach versehentlich in die Vagina appliziertem Kontrastmitteleinlauf. $Z$. Rechtsmed. 86 (1980), 71.

3 Geipel, A.: Tödlicher Zwischenfall nach Kontrastmitteleinlauf des Mastdarmes. Dtsch. Z. ges. gerichtl. Med. 59 (1967), 255.

4 Mattig, W., E. Lignitz: Der Einlauf - eine ungefährliche medizinische Technik? In Walther, G., H. Th. Haffner (Hrsg.): Festschrift für Horst Leithoff (Kriminalistikverlag: Heidelberg 1985), 613.

5 Mattig, W., E. Lignitz, J. Eulitz, A. K. Schmauss: Fehler und Gefahren bei Darmeinläufen. Heilberufe 37 (1985), 218.

6 Tschirren, B.: Der Narkosezwischenfall (Huber: Bern-StuttgartToronto 1987).

Dr. H. T. Haffner, Dr. M. Graw Institut für Gerichtliche Medizin der Universität

Nägelestr. 5

W-7400 Tübingen 1 